Check for updates

Cite this: J. Mater. Chem. A, 2019, 7, 1244

Received 19th October 2018 Accepted 6th December 2018

DOI: $10.1039 / \mathrm{c} 8 \mathrm{ta} 10057 \mathrm{~h}$

rsc.li/materials-a

\section{Self-floating hybrid hydrogels assembled with conducting polymer hollow spheres and silica aerogel microparticles for solar steam generation $\uparrow$}

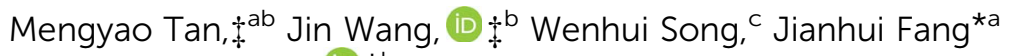 \\ and Xuetong Zhang (D) *bc
}

\begin{abstract}
The design and synthesis of solar steam generation materials have received considerable attention due to their capacity to produce freshwater from seawater or contaminated water by a straightforward utilisation of solar energy. The practical application of these materials, however, is restricted by their low evaporation efficiency and non-durable floating capacity on water. Herein, flexible and self-floating polyvinyl alcohol (PVA) based hybrid hydrogels for solar steam generation are designed and synthesized by assembling two types of functional particles within the network: conducting polymer hollow spheres (CPHSs) for achieving solar absorption and heat conversion, and silica aerogel microparticles for density reduction and efficient energy conversion confined to a small amount of surrounding water. Following a freezing process, functionalized hybrid hydrogels with macro-sized channels are generated, contributing to rapid water supply. The Janus surface nature, with one side being hydrophilic (contact angle ca. 60 ${ }^{\circ}$ ) and another hydrophobic (contact angle up to $135^{\circ}$ ), of the hybrid hydrogel was found due to the formation of a gradient distribution of silica aerogel particles via controlling the gelation conditions. Consequently, the density of the hybrid hydrogels is controlled in the range of $0.8-1.0 \mathrm{~g} \mathrm{~cm}^{-3}$ and the thermal conductivity of the corresponding xerogels in the range of $0.030-0.035 \mathrm{~W} \mathrm{~m}^{-1} \mathrm{~K}^{-1}$, depending on the content of the silica aerogels. High water production of the hybrid hydrogel at a rate of $1.83 \mathrm{~kg} \mathrm{~m}^{-2} \mathrm{~h}^{-1}$ under 1 sun illumination has been demonstrated, which is an important step towards a cost-effective solution for the scarcity of clean water.
\end{abstract}

\section{Introduction}

Solar energy is a promising source for boosting the evolution of sustainable and renewable energy technologies. Solar-to-electric conversion systems, such as perovskite solar cells, ${ }^{1,2}$ dyesensitized solar cells, ${ }^{3,4}$ and organic solar cells, ${ }^{5-9}$ and solar-tothermal conversion processes, such as domestic heating, ${ }^{10}$ water desalination and remediation, ${ }^{11-13}$ have received considerable attention from both the academic and industrial community. Among the solar energy applications, solar steam generation has attracted increasing interest because freshwater could be directly produced from polluted or saline water.

\footnotetext{
aDepartment of Chemistry, College of Sciences, Shanghai University, Shanghai 200444, P. R. China. E-mail: jhfang@shu.edu.cn

${ }^{b}$ Suzhou Institute of Nano-tech and Nano-bionics, Chinese Academy of Sciences, Suzhou 215123, P. R. China. E-mail: xtzhang2013@sinano.ac.cn

'Department of Surgical Biotechnology, Division of Surgery \& Interventional Science, University College London, London, NW3 2PF, UK. E-mail: xuetong.zhang@ucl.ac.uk $\dagger$ Electronic supplementary information (ESI) available: Photos of the functional hydrogels, extra SEM and TEM of the CPHSs, aerogels, and hydrogels, water transport rate, saturated water content, evaporation of the hydrogels, etc. See DOI: $10.1039 / \mathrm{c} 8 \mathrm{ta} 10057 \mathrm{~h}$

\$ These authors contributed equally.
}

Though significant progress has been made in enhancing solar mediated water evaporation, many challenges remain to be overcome, mainly in the following areas: (1) enhancing the solar absorption and improving light-heat conversion efficiency; (2) confining the converted energy to a small amount of water; (3) ensuring sustainable and rapid water supply to the localized heated area; and (4) synthesizing materials with long life span and capable of self-floating on water.

To tackle these problems, great efforts have been made through development and application of new functional materials. For instance, ultra-black semiconductors, ${ }^{14}$ plasmonic nanoparticles, ${ }^{15-17}$ and nanocarbon materials ${ }^{18-21}$ have been investigated for enhancing solar absorption. Carbon-based materials, ${ }^{22,23}$ conducting polymers, ${ }^{24}$ bio-inspired structures, ${ }^{25,26}$ and aerogels ${ }^{27-30}$ have been used to improve energy confinement by heat localization. Freezing and carbonization have been applied to produce large channels for rapid water supply, ${ }^{31-33}$ and superhydrophobic modification was used to fabricate self-floating membranes ${ }^{34}$ for solar steam generation. Despite the improvement, more functional and robust materials and devices based on them are still desirable to solve all the challenges. Interestingly, aerogels are a kind of highly porous material possessing extremely low density and low 
thermal conductivity, and they have been used as either bulk or powdery materials in the fields of thermal insulation, superhydrophobic modification, catalyst support, etc. ${ }^{35-41}$ By taking these advantages, it is hypothesised that, as illustrated in Fig. 1a, if aerogel microparticles can be incorporated in a device for solar steam generation, the product will be light weight and self-floating on water, and the heat may be confined by the aerogels for localized heating. To prove this hypothesis, herein, a series of energy-converting composite hydrogels based on polyvinyl alcohol (PVA) were designed and synthesized, which could self-float on water. Conducting polymer hollow spheres $(\mathrm{CPHSs})^{42-44}$ were used as a robust solar energy absorption and light-heat conversion agent, while superhydrophobic silica aerogel microparticles were applied to afford a hydrophobic upper surface with further weight reduction, and thus the hybrid hydrogels were proven to be self-floating on water so that the water could be efficiently evaporated. Finally, freeze-andthaw treatment of the hydrogels was performed in order to produce macro-sized channels within the hydrogel network for more rapid water supply. A unique macro-sized Janus surface hydrogel with a hydrophilic bottom layer (contact angle $c a .60^{\circ}$ ) and a hydrophobic top layer (contact angle up to $135^{\circ}$ ) was
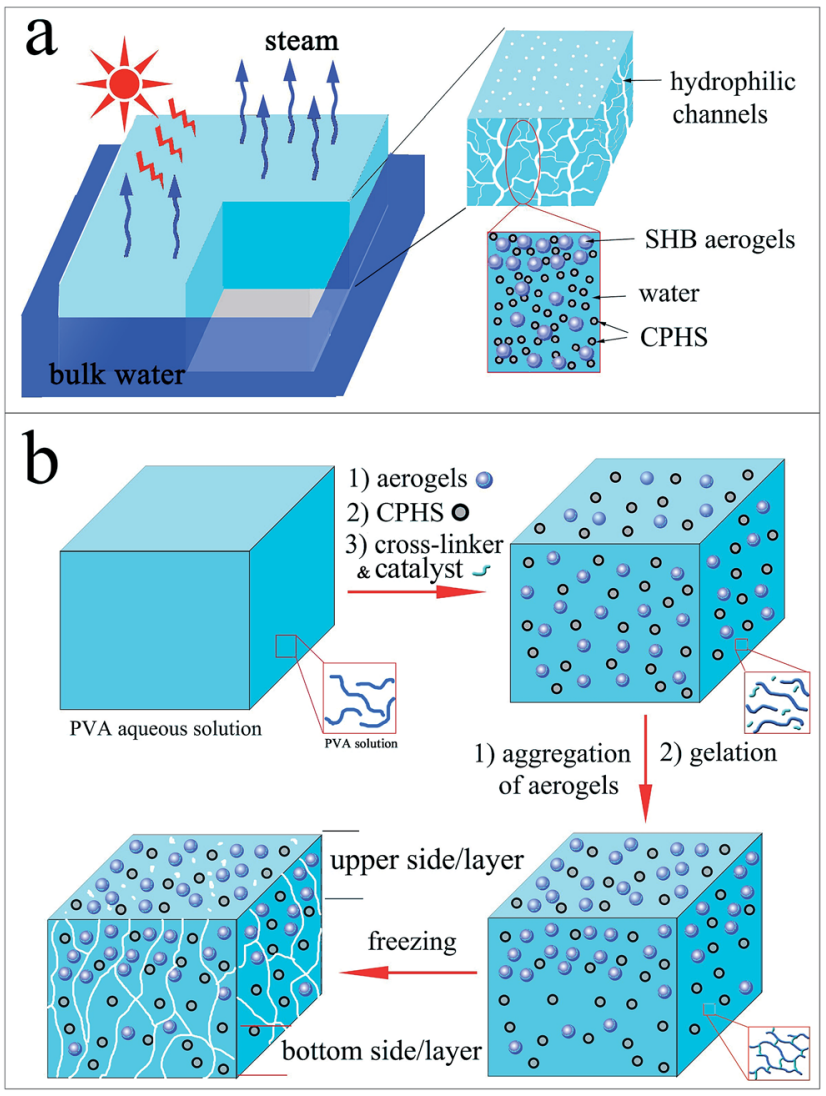

Fig. 1 (a) The proposed functional CPHS/aerogel/PVA hybrid hydrogels for solar steam generation: hydrophilic channels are introduced by freeze-and-thaw cycles and used for rapid water supply; CPHSs are used as a solar energy absorbent, and aerogel microparticles for reducing density and thermal conductivity. (b) Schematic diagram of the synthetic approach for producing the CPHS/aerogel functionalized hydrogels. achieved via controlling the distribution of aerogel microparticles across the thickness of the membrane under different gelation conditions. As a result, a high water-production rate of $1.83 \mathrm{~kg} \mathrm{~m}^{-2} \mathrm{~h}^{-1}$ under 1 sun illumination $\left(1 \mathrm{~kW} \mathrm{~m}^{-2}\right)$ has been achieved. The densities of the hybrid hydrogels were in the range of $0.85-1.0 \mathrm{~g} \mathrm{~cm}^{-3}$ and the thermal conductivity of the corresponding xerogels in the range of $0.030-0.035 \mathrm{~W} \mathrm{~m}^{-1} \mathrm{~K}^{-1}$, depending on the amount of silica aerogels.

\section{Experimental}

\section{Materials}

PVA with an alcoholysis degree of $98-99 \%\left(\mathrm{~mol} \mathrm{~mol}^{-1}\right)$, pyrrole, aniline, $\mathrm{HCl}(37 \%)$, glutaraldehyde, and ammonium persulfate (APS) were purchased from Sigma-Aldrich. Triton X-100 (TX100) was purchased from Beijing Chemical Works. Aniline and pyrrole were distilled under reduced pressure before use. De-ionized water was used in all the experiments. Poly(anilineco-pyrrole) hollow spheres (CPHSs) were prepared according to the literature, ${ }^{42-44}$ and the TEM morphology of the CPHSs is shown in Fig. S1 (ESI†). Hydrophobic $\mathrm{SiO}_{2}$ aerogel microparticles (see Fig. S2 for the SEM images in the ESI $\dagger$ ) were synthesized according to our previous work, the contact angle is $149^{\circ}$ and the BET surface area is $576 \mathrm{~m}^{2} \mathrm{~g}^{-1}$ (Fig. S3, ESI $\uparrow$ ). ${ }^{45,46}$ All other reagents were used without further purification.

\section{Preparation of hybrid hydrogels containing CPHSs and silica aerogel microparticles}

In a typical process, $8 \mathrm{mg} \mathrm{SiO}_{2}$ aerogel microparticles were added to $8 \mathrm{~g}$ PVA aqueous solution (10 wt\%), followed by the addition of $2 \mathrm{ml}$ of PPy-PANI hollow sphere aqueous solution (60 $\mathrm{mg} \mathrm{ml}^{-1}$ ). The components were mixed by vigorous stirring. Subsequently, $400 \mu \mathrm{l}$ of $\mathrm{HCl}(1 \mathrm{M})$ serving as the catalyst and 192 $\mu \mathrm{l}$ of glutaraldehyde $(10 \mathrm{wt} \%)$ serving as the crosslinker were added to the solution. The mixture was kept at $20^{\circ} \mathrm{C}$ and the hydrogel formed in $2 \mathrm{~h}$. The obtained PVA hydrogel was washed with DI water. The purified gel was directionally frozen by liquid nitrogen from the bottom side and then thawed in DI water at a temperature of $30{ }^{\circ} \mathrm{C}$. The freeze-and-thaw process was repeated 10 times.

\section{Characterization}

The morphology of the samples was characterized using a scanning electron microscope (SEM) (S-4800) operated at 10 $\mathrm{kV}$ and a transmission electron microscope (TEM, Tecnai G2 F20 S-Twin) operated at $200 \mathrm{kV}$. An infrared camera was used to take infrared photographs. The thermal conductivity at room temperature was measured using a transient hot wire method (XIATECH TC3000, China); the data were collected three times with a $5 \mathrm{~min}$ interval between each measurement. Water contact angles were measured using a contact angle meter (DSA 100, KRUSS Corporation, Germany). The dynamic rheological measurements were carried out on a Physica MCR301 apparatus at $25^{\circ} \mathrm{C}$. The storage modulus $\left(G^{\prime}\right)$ and loss modulus $\left(G^{\prime \prime}\right)$ of the hydrogels were measured at a frequency ranging from 0.1 to $10 \mathrm{~Hz}$ with a strain amplitude of $100 \mathrm{~Pa}$ at $27^{\circ} \mathrm{C}$. The optical 
transmittance and reflectance spectrum of the CPHS hybrid hydrogel was recorded in the range of 200-2000 $\mathrm{nm}$ with a Shimadzu UV3600 spectrophotometer attached to an integrating sphere (ISR-3100). The absorption efficiency was then calculated using $A=1-R-T$, where $R$ and $T$ are the reflection and transmission efficiencies, respectively.

\section{Water evaporation performance of the hydrogels}

The steam generation experiments were performed with a solar simulator (Newport 94043A) and the light intensity was kept at 1 $\mathrm{kW} \mathrm{m}{ }^{-2}$ (1 sun). All the test data were obtained at an ambient temperature of $22 \pm 2{ }^{\circ} \mathrm{C}$ and a relative humidity of $\approx 48 \%$. The hybrid hydrogels with a diameter of $3.5 \mathrm{~cm}$ and thickness from 2 to $7 \mathrm{~mm}$ were floated on water in a cylindrical polystyrene (PP) container with a diameter of the water surface of $c a .3 \mathrm{~cm}$ for solar steam generation. The mass change during water evaporation was measured using a high-accuracy balance (Quintix 224-1CN, $0.1 \mathrm{mg}$ accuracy). All evaporation rates were measured after stabilization under 1 sun for 60 minutes.

The corresponding energy efficiency $(\eta)$ for solar to vapor generation can be calculated using the following formula:

$$
\eta=\dot{m} h_{\mathrm{v}} / C_{\mathrm{opt}} P_{0}
$$

in which $\dot{m}$ is the mass flux, $h_{\mathrm{v}}$ is the vaporization enthalpy of the water in the hydrogels, $P_{0}$ is the solar irradiation power of one sun $\left(1 \mathrm{KW} \mathrm{m}^{-2}\right)$, and $C_{\text {opt }}$ refers to the optical concentration on the absorber surface.

\section{Results and discussion}

Due to their high water contents (up to $80 \mathrm{wt} \%$ ) and highly porous structures, hydrogels have been recently adopted as ideal matrices for solar steam generation. ${ }^{32,33}$ However, due to their higher densities than water, it's difficult for them to float on water for a long time. In this work, a simple and facile approach to the design and production of self-floating composite hydrogels with densities lower than $1 \mathrm{~g} \mathrm{~cm}^{-3}$ was developed by the introduction of superhydrophobic silica aerogel microparticles (SAMs). For instance, a PVA composite hydrogel containing $15 \mathrm{wt} \%$ SAMs was achieved. Due to its low density $\left(0.5 \mathrm{~g} \mathrm{~cm}^{-3}\right)$, it could stably self-float on water for 1 year (see Fig. S4, ESI $\dagger$ ). However the composite PVA hydrogels with high content of SAM were hydrophobic inside, which is disadvantageous for a sustainable and rapid water supply in the local heated area. Thus, a low silica aerogel content ranging from 0.08 to 0.4 wt $\%$ was applied in this work. Following the approach illustrated in Fig. 1b, a mixture of aerogels, CPHSs, a cross-linker and a catalyst with aqueous PVA was kept at $20^{\circ} \mathrm{C}$ to slow down the gelation process to provide enough time for aggregation and migration of the SAMs towards the upper side of the composite hydrogels. After gelation for $2 \mathrm{~h}$, the obtained functional hydrogels appeared black with the rougher upper surface of the hydrogels being hydrophobic (with a contact angle of $c a .135^{\circ}$ ), and the smoother bottom surface of the hydrogels being hydrophilic (with a contact angle of $c a .60^{\circ}$ ), as shown in Fig. $2 \mathrm{a}$ and $\mathrm{b}$. The results indicated that a phase separation occurred during the gelation process and more SAMs might aggregate and migrate towards the upper layer of the hydrogels, which is confirmed by the SEM-EDS results in the following section. As expected, the hybrid hydrogel was light weight $\left(0.85-1.0 \mathrm{~g} \mathrm{~cm}^{-3}\right)$ and self-floating on water (Fig. S5 ESI $\dagger$ ), and the low density of the hydrogel may be ascribed to the aerogels and indicated that water did not fill in the pores of aerogels due to their hydrophobicity. When the hydrogels were used for solar steam generation, the bottom side of the hydrogels was partially submerged in water while the upper side was exposed to air. If the hydrogel membrane was put the other way around, by submerging the upper side in water, the hydrophobic nature of the upper surface may play an opposite role of prohibiting the sustained water supply.

The viscoelasticities of the hydrogels in terms of storage modulus, $G^{\prime}$, and loss modulus, $G^{\prime \prime}$, as function of frequency under shearing are shown in Fig. 2c. The dynamic rheological frequency sweep of the hydrogels shows a stable storage modulus, $G^{\prime}$, and loss modulus, $G^{\prime \prime}$, with little dependence on the range of frequency tested. The $G^{\prime}$ value is significantly higher than the $G^{\prime \prime}$ value (almost zero) in all cases, indicating dominance of the elastic behaviour with little energy loss at small strain owing to their chemically crosslinked polymeric network. The high elasticity of the hydrogels was also confirmed by a static mechanical test, with a recoverable compression strain of more than $60 \%$ (Fig. S6 ESI $\dagger$ ). The hydrogels could be reversibly distorted into various shapes (Fig. S7 ESI $\dagger$ ).

SEM images in Fig. 2d-f show the morphology of the dehydrated hydrogels. As seen from the cross-section image (Fig. 2d), there are a number of macro-sized channels (from several to hundreds of micrometers) in the vertical direction, which resulted from the directional freeze-and-thaw treatments of the hydrogels. Besides, there are also various interconnected micro-pores with size lower than $10 \mu \mathrm{m}$ (Fig. 2e and f) on the walls of large channels. These structures were envisaged to facilitate water transport. ${ }^{31-33}$ As shown in Fig. S8 (ESI $\dagger$ ), all the functional hydrogels possessed high saturated water contents $\left(Q_{\mathrm{s}}\right)$, e.g. 3.63, 3.55, 3.24, and $2.86 \mathrm{~g} \mathrm{~g}^{-1}$ for the hydrogels with aerogel contents of $0.08,0.16,0.24$, and $0.4 \mathrm{wt} \%$, respectively, indicating that the $Q_{\mathrm{s}}$ can be tuned with the amount of silica aerogels. In addition, the water transport in the hydrogels was evaluated by the dynamic analysis of their swelling process. The swollen time from the half-saturated state $\left(0.5 Q_{\mathrm{s}}\right)$ to the saturated state $\left(Q_{\mathrm{s}}\right)$ can reveal the water transport in the hydrogels. ${ }^{32}$ The water transport rate $(V)$ can be calculated using

$$
V=0.5 Q_{\mathrm{s}} / t
$$

where $t$ is the half-swollen time. As shown in Fig. S9 (ESI $\dagger$ ), with one gram of the freeze-dried hydrogel (xerogel), the hydrogels show a $V$ of $0.030,0.026,0.019$, and $0.017 \mathrm{~g} \mathrm{~min}^{-1}$, indicating the tunable water transport properties of the functional hydrogels.

The SEM-EDS 2D images of the hydrogels (Fig. $2 \mathrm{~g}$ and $\mathrm{h}$ ) show that carbon and nitrogen elements were homogeneously distributed, which indicated that the CPHSs were well dispersed in the hydrogels (the nitrogen was from the CPHSs). However, 

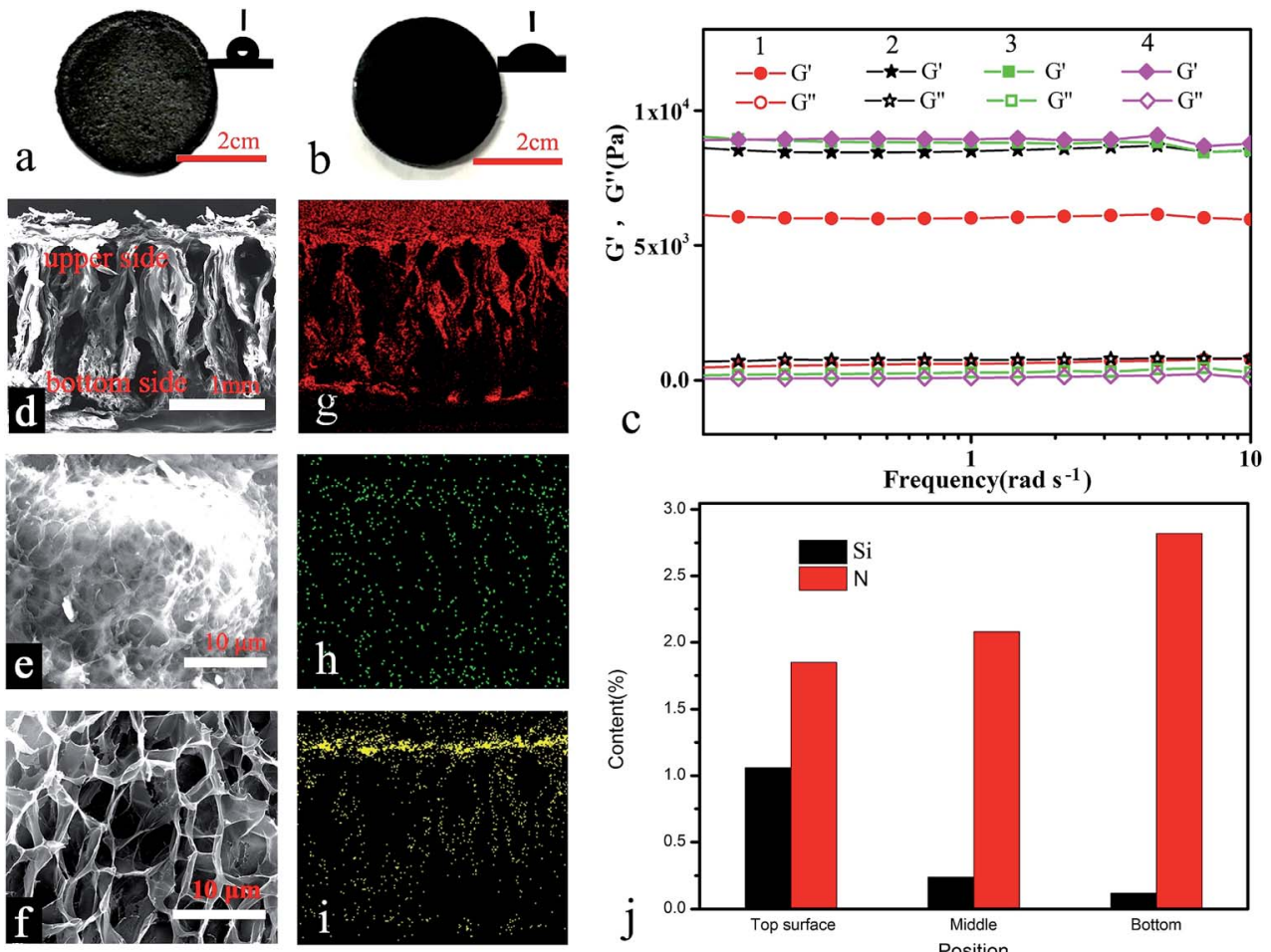

Fig. 2 Photo images of the (a) upper surface and (b) bottom surface of the CPHS/aerogel functionalized hydrogel, inset is the contact angle image; (c) dynamic shear rheology behaviours of the hydrogels, the curves of storage modulus $\left(G^{\prime}\right)$ and loss modulus $\left(G^{\prime \prime}\right)$ vs. frequency, samples 1-4 correspond to the hydrogels with CPHS contents of 0.4, 0.8, 1.2, and 1.6 wt\%; SEM images of the hydrogels: (d) cross-section of the hydrogel, (e) upper surface of the hydrogel, and (f) bottom surface of the hydrogel; SEM-EDS 2D images of the cross-section of the hydrogel: (g) carbon, (h) nitrogen, and (i) silicon; (j) Si and $\mathrm{N}$ contents of different parts of the hydrogel from SEM-EDS spectra.

the signal of silicon was significantly stronger in the upper side of the hydrogel, which indicated that the silica aerogels were aggregated and migrated towards the top of the hydrogel, possibly due to the low density of the aerogels $\left(c a .50 \mathrm{mg} \mathrm{cm}^{-3}\right)$ and their non-wettability by water. The distribution of Si and $\mathrm{N}$ across the membrane thickness was quantified using the SEMEDS spectra, as shown in Fig. 2j, with the content of silica (corresponding to the silica aerogels) significantly decreasing from $1 \mathrm{wt} \%$ in the top layer to $0.1 \mathrm{wt} \%$ in the bottom layer. The SEM image of the top layer of the hydrogel in Fig. 2e and S10 $\mathrm{ESI} \dagger$ further indicated that the aerogel microparticles were enriched on the top surface of the hydrogel and remained intact as the porous structure could be clearly observed, while they were almost unobservable in the bottom layer of the hydrogel (see Fig. $2 \mathrm{f}$ and S11, ESI $\dagger$ ). The gradient distribution of the aerogels may explain the reason for the Janus surface nature of the hydrogel (with a hydrophobic top layer and a hydrophilic bottom layer). Opposed to Si content, the content of nitrogen, which corresponds to CPHSs, was increased from 1.8 to $2.7 \mathrm{wt} \%$ from the top to the bottom of the hydrogel (Fig. 2j). Such an inverse dual-gradient hybrid structure is prone to provide multiple functionalities to the hydrogels, which is envisaged to have an influential role in the solar steam generation performance, as systematically investigated in the following section.

Functional hydrogels with different contents of CPHSs, silica aerogels, and thickness were synthesized and investigated for solar steam generation. An impressive evaporation rate up to $1.83 \mathrm{~kg} \mathrm{~m}^{-2} \mathrm{~h}^{-1}$ with a high solar energy conversion efficiency of $82.2 \%$ had been obtained in the case of the hydrogel with a thickness of $5 \mathrm{~mm}$, and $1.2 \mathrm{wt} \%$ and $0.08 \mathrm{wt} \%$ CPHSs and aerogels, respectively. The resulting hydrogel could self-float on water (Fig. 3a) and its temperature increased rapidly under 1 sun solar illumination (Fig. $3 \mathrm{~b}-\mathrm{d}$ ). The temperatures of the hydrogel and the bulk water were real-time monitored. As presented in Fig. 3e, the temperature of the top surface increased from ambient temperature $\left(23.3{ }^{\circ} \mathrm{C}\right)$ to $33{ }^{\circ} \mathrm{C}$ in several minutes and finally up to $40{ }^{\circ} \mathrm{C}$, and the temperatures inside the hydrogel and at the bottom surface of the hydrogel also significantly increased to $35^{\circ} \mathrm{C}$. In comparison, the temperature of the bulk water slowly increased and was lower than $30^{\circ} \mathrm{C}$ even after $1 \mathrm{~h}$. The results indicated that heat transportation from the hydrogel to the bulk water was limited, possibly due to the low thermal conductivity of the hydrogel (around 0.20 and $0.54 \mathrm{~W} \mathrm{~m}^{-1} \mathrm{~K}^{-1}$ for the upper side and the bottom side, respectively, Fig. S12 ESI $\dagger$ ), thanks to the presence of silica aerogels. Besides, it could be seen that the temperature of the top side of the hydrogel rapidly increased to $35^{\circ} \mathrm{C}$ in $10 \mathrm{~min}$ and then reached a balance. The result may indicate that the lightto-heat conversion and the water evaporation reach equilibrium in $10 \mathrm{~min} .{ }^{32} \mathrm{Fig}$. $3 \mathrm{f}$ shows the water evaporation rate (ER) of the hydrogel under 1 sun and in the dark. The ER values of pure water under similar conditions are also presented. First, 

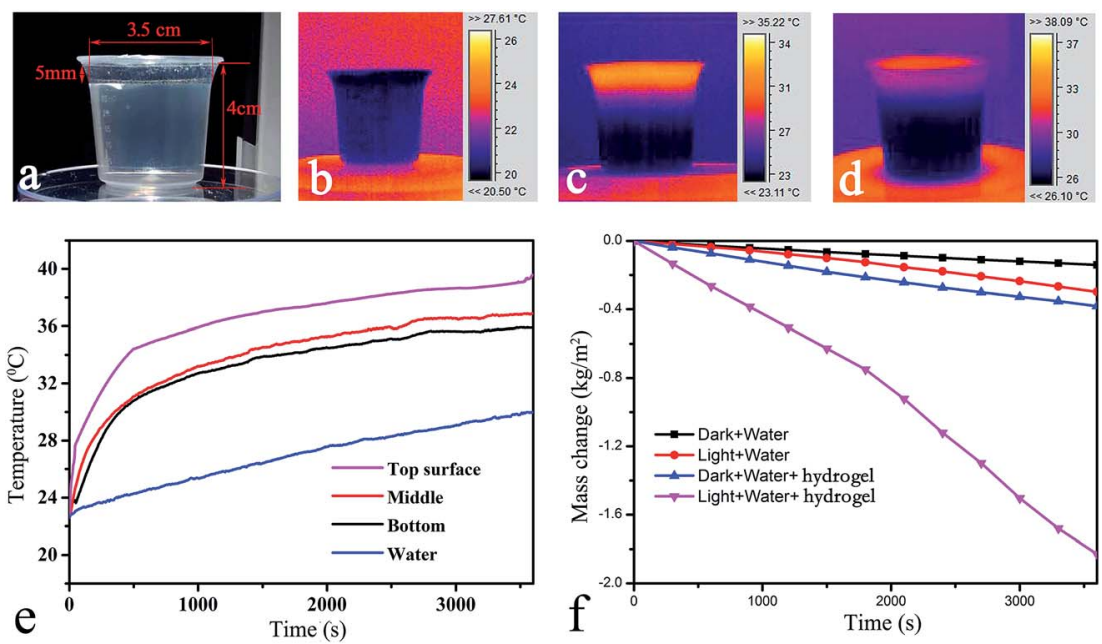

Fig. 3 (a) Photo of the solar steam generation measurement under 1 sun illumination: infra-red images of the functional hydrogels kept under 1 sun illumination for: (b) $0 \mathrm{~min}$, (c) $30 \mathrm{~min}$, and (d) $60 \mathrm{~min}$; (e) temperature-time curves of different parts of the functional hydrogel and bulk water under 1 sun illumination; ( $f$ ) evaporation rate of water through the hydrogels under different conditions (in the dark or under 1 sun), in the presence/absence of hydrogels as indicated.

the ER of pure water in the absence of hydrogel was investigated, and the ER of water was 0.14 and $0.32 \mathrm{~kg} \mathrm{~m}^{-2} \mathrm{~h}^{-1}$ in the dark and under 1 sun, respectively. Impressively, the ER of water in the presence of the hydrogel reached $1.83 \mathrm{~kg} \mathrm{~m}^{-2} \mathrm{~h}^{-1}$ under 1 sun, a remarkable increment of 5.7 times. The ER value of the hydrogel in the dark only slightly increased to $0.38 \mathrm{~kg} \mathrm{~m} \mathrm{~m}^{-2} \mathrm{~h}^{-1}$, possibly due to the absorption of infrared radiation from the surrounding environment. As confirmed by the absorption spectra of the hydrogel shown in Fig. S13 ESI, $\dagger$ the averaged absorption is $90 \%$ across $200-2000 \mathrm{~nm}$. It should be pointed out that in all the experiments, the bottom side of the hydrogels was submerged in water unless it is pointed out, because the bottom side of the hydrogels is hydrophilic, which facilitated water transportation to the hydrogels and resulted in higher ER values (as illustrated in Fig. S14, ESI†).

In comparison, if no silica aerogels were introduced, the CPHS functionalized hydrogels would sink into water (Fig. S15, ESI†). Regardless of the presence of CPHSs as a photothermal conversion agent, water could not be evaporated through the hydrogel, and the ER in this case was only $0.74 \mathrm{~kg} \mathrm{~m}^{-2} \mathrm{~h}^{-1}$ (Fig. S16, ESI $\dagger$ ). On the other hand, if no CPHSs were introduced, the aerogel functionalized hydrogel could self-float on water (Fig. S17, ESI†). However, due to the lack of a photothermal conversion agent (CPHSs), the ER in this case was even more lower, only $0.56 \mathrm{~kg} \mathrm{~m}^{-2} \mathrm{~h}^{-1}$ (Fig. S16, ESI $\dagger$ ). Besides, the superior performance of the hollow spheres over the solid spheres was also confirmed by parallel experiments. Solid microspheres based on polypyrrole (PPy), polyaniline (PANI), and PPy-PANI copolymers were prepared according to the literature $^{43,47}$ (Fig. S18, ESI $\dagger$ ). The ER values of the hydrogels derived from PPy/aerogel, PANI/aerogel, PPy-PANI/aerogel, and CPHS/aerogel were measured and the values were 1.0, 1.19, 1.22 , and $1.47 \mathrm{~kg} \mathrm{~m}^{-2} \mathrm{~h}^{-1}$, respectively, as shown in Fig. S19, ESI. $\dagger$ Clearly, the results confirmed that much higher ER values could be obtained when hollow spheres were introduced into the hydrogel, in that vapor bubbles might have been simultaneously generated from the interior and exterior of the nanoshell of the hollow spheres, thus minimising light scattering and enhancing light absorption. ${ }^{48}$ If CPHSs alone were used for solar steam generation, the microspheres were quickly wetted by water and they sank in water (Fig. S20, ESI $\dagger$ ), and an ER value of $0.77 \mathrm{~kg} \mathrm{~m}^{-2} \mathrm{~h}^{-1}$ was obtained (Fig. S21, ESI $\dagger$ ), which is almost the same as that of the hybrid hydrogel without the silica aerogels (Fig. S16, ESI $\dagger$ ). The reason may also be that water evaporation did not occur directly from the CPHSs, and the bulk water was heated up (the heating of the water was not localized, see Fig. S22, ESI†).

Apart from the aforementioned parameters, the contents of the CPHSs and aerogels, and hydrogel thickness may also greatly impact the ER values of the hydrogels. To optimise the formulation and structure of the hydrogel, the effects of the variation of functional components and thickness of the hydrogel on the evaporation capacities of the hydrogels were investigated, and are presented in Fig. 4. As seen in Fig. 4a, the ER values increased with the increment of CPHS content, and reached a maximum when the content of CPHSs was $1.2 \mathrm{wt} \%$. Further increasing the CPHS content did not increase the ER. The effect of silica aerogels on the ER is shown in Fig. $4 \mathrm{~b}$. The lowest content of aerogels, $0.08 \mathrm{wt} \%$, appeared to contribute to the highest evaporation capacity of the hydrogel. Further increasing the amount of aerogels resulted in lower ERs, possibly due to a trade-off effect of increasing hydrophobicity, which reduced both the saturated water content in the hydrogel and the water transportation rate, as confirmed in the above section, Fig. S9, ESI. $\dagger$ It should be pointed out that bulk aerogels have been used for solar steam generation; ${ }^{27-30}$ however, their ERs were relatively low (0.9-1.4 $\left.\mathrm{kg} \mathrm{m}^{-2} \mathrm{~h}^{-1}\right)$ and the conversion efficiencies were lower than $75 \%$ under 1 sun illumination. The possible reason may be that the aerogels were filled with water when they were used for steam generation, in which they 

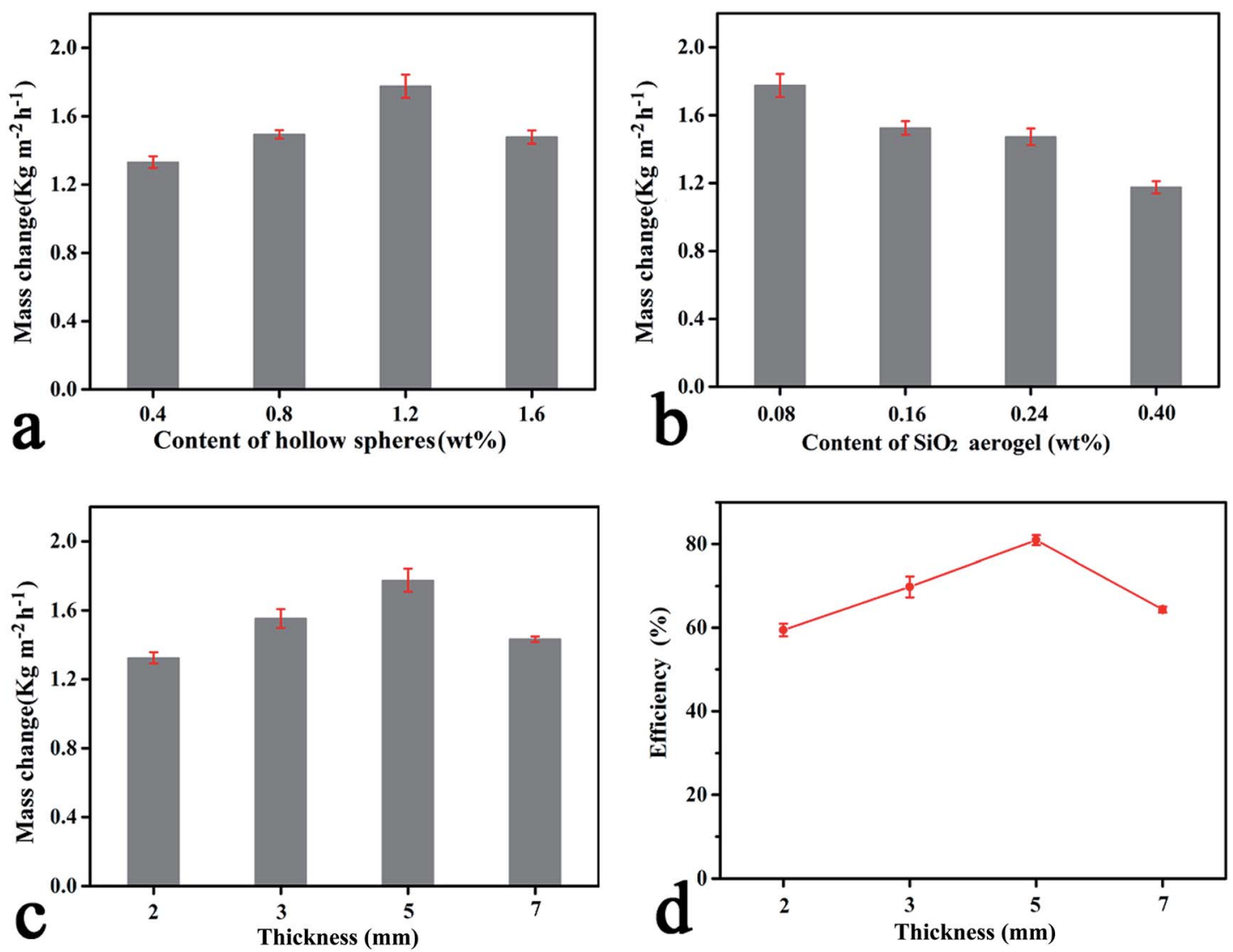

Fig. 4 (a) ER values related to the content of hollow spheres of the hydrogels with the same thickness (5 mm) and silica aerogel content $(0.08 \mathrm{wt} \%)$. (b) ER values related to the content of silica aerogels of the hydrogels with the same thickness (5 mm) and hollow sphere content (1.2 wt\%). (c) ER values related to the thickness of the hydrogels with the same aerogel content (0.08 wt\%) and hollow sphere content (1.2 wt\%). (d) Solar energy conversion efficiency of the hydrogels with different thicknesses, consisting of 0.08 wt $\%$ silica aerogels and 1.2 wt $\%$ hollow spheres.

became hydrogels and the excellent thermal insulation properties of the aerogels were decreased. But in our work, the aerogels remained intact as confirmed by their low densities and SEM images (Fig. S10, ESI $\dagger$ ). Based on the optimised composition of the hydrogel, the effect of thickness of the hydrogel on the evaporation rate was also evaluated, as shown in Fig. 4c, with the ER value peaking at a thickness of $5 \mathrm{~mm}$. If the hydrogel was too thin, it could easily roll up under illumination. If the hydrogel was too thick, water transportation rate also reduced due to a longer diffusion distance. The corresponding solar energy conversion efficiency showed a similar tendency as shown in Fig. $4 \mathrm{~d}$, reaching $82.2 \%$ when the thickness was $5 \mathrm{~mm}$, with optimised CPHS and aerogel contents of $1.2 \mathrm{wt} \%$ and $0.08 \mathrm{wt} \%$, respectively.

The freeze-and-thaw process, which introduced macro-sized channels in the vertical direction in the hybrid hydrogels, could also significantly increase the ER value. In contrast, there is no porous structure in the hydrogels without freeze-and-thaw treatment (Fig. S23, ESI $\dagger$ ), and the ER value of the corresponding hydrogel was $1.27 \mathrm{~kg} \mathrm{~m}^{-2} \mathrm{~h}^{-1}$ (Fig. S24, ESI $\dagger$ ). The results indicate that introducing a porous structure in the hydrogel by freeze-and-thaw is an efficient approach to improve its steam generation efficiency. ${ }^{33}$
Furthermore, the high ER and conversion efficiency of the functional hybrid hydrogels could be repeatedly achieved for 30 cycles with no sign of decline, indicating their high stability as shown in Fig. 5a. The repeat test of the hydrogels was conducted each day for 2 hours. In fact, the hydrogel could float on water for 6 months, and there was no sign of sinking into the water, which was ascribed to the presence of superhydrophobic silica aerogels in the hydrogel matrix. Based on these results, the working mechanism of the multi-functional hydrogels for solar steam generation is proposed as illustrated in Fig. 5b. Firstly, the CPHSs distributed within the PVA hydrogel network play an essential role in transforming solar energy into thermal energy. The localized water around the CPHSs heated up and the evaporation rates were elevated. Secondly, the aerogels afford the self-floating capacity to the hydrogels, providing an interface between the hydrogel and air, so that the vapor could be directly transferred to the environment and collected. Besides, the aerogels may significantly decrease the thermal conductivity of the hydrogels, and thus the heat converted by the CPHSs could be well confined to their surrounding water. Thirdly, vertically aligned macro-sized channels induced by freeze-andthaw cycles facilitate water transport into the network towards the upper surface of the hydrogel. As a consequence, such 

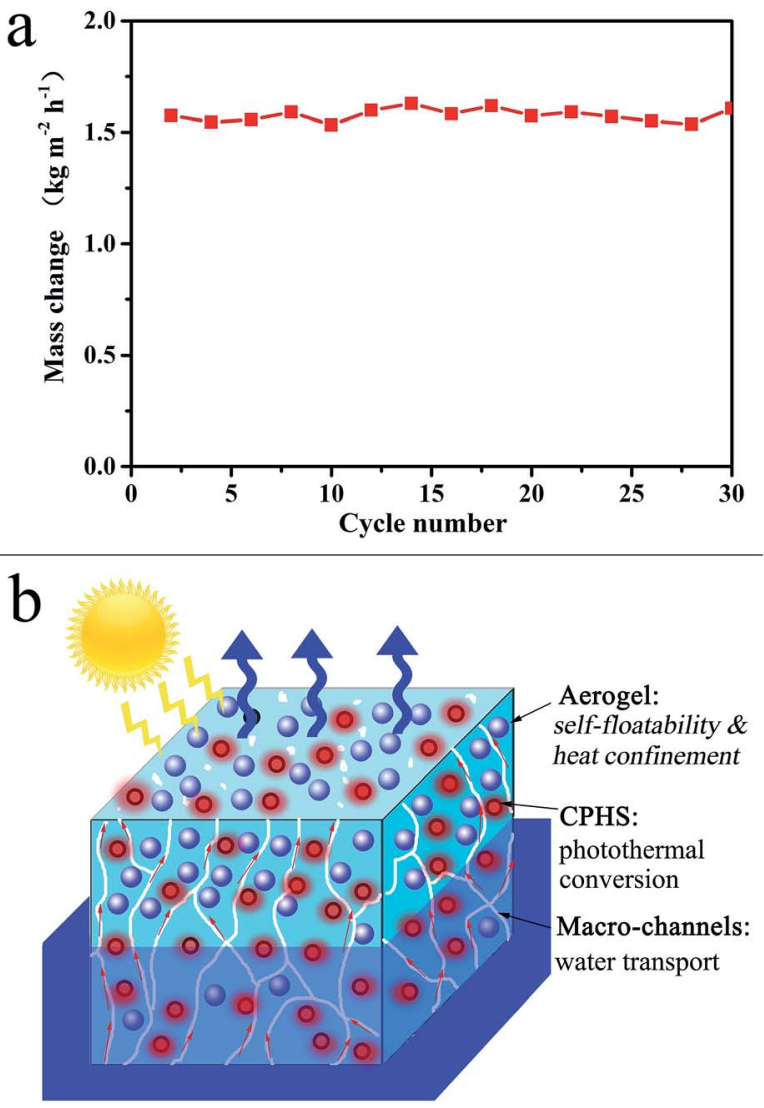

Fig. 5 (a) Reusability of hydrogels under 1 solar irradiation for 30 cycles, each cycle was tested for $2 \mathrm{~h}$. (b) Schematic illustration of solar steam generation based on the functional hybrid hydrogels.

a coherent assembly of a heterogenetic structure offers an appealing ER of $1.83 \mathrm{~kg} \mathrm{~m}^{-2} \mathrm{~h}^{-1}$, which is among the highest values in the field. ${ }^{11-13}$

\section{Conclusion}

In summary, a series of CPHS and aerogel functionalized hybrid hydrogels were designed and synthesize for rapid solar steam generation. The desirable functions of solar steam generation materials, e.g. high photothermal conversion efficiency (up to $82.2 \%$ ), self-floatability, low heat transfer to the surrounding environment, a fast water transport rate (up to $0.03 \mathrm{~g} \mathrm{~min}^{-1}$ ), and a high ER of the hydrogel (up to $1.83 \mathrm{~kg} \mathrm{~m}^{-2} \mathrm{~h}^{-1}$ ), have been achieved in an optimised functional hydrogel, contributed by a unique assembled heterogenetic structure of a 3D network consisting of an inverse dual-gradient distribution of CPHSs, silica aerogels, and vertical macro-channels across the membrane thickness. The obtained hydrogels were robust, flexible and could self-float on water, with an upper hydrophobic and bottom hydrophilic Janus surface. The proven concept of introducing energy concerting particles and hydrophobic aerogel microparticles with an inverse-dual gradient distribution into a hydrophilic hydrogel matrix may lead to fabrication of various new families of functional hydrogels that could self-float on water, and may be an important step towards a solution for the scarcity of clean water.

\section{Conflicts of interest}

There are no conflicts to declare.

\section{Acknowledgements}

This work was financially supported by the National Natural Science Foundation of China (51572285 and 51773225), the National Key Research and Development Program of China (2016YFA0203301), the Royal Society Newton Advanced Fellowship (NA170184) and the Natural Science Foundation of Jiangsu Province (BK20170428).

\section{References}

1 S. Chatterjee and A. J. Pal, J. Mater. Chem. A, 2018, 6, 37933823.

2 Z. Fan, K. Sun and J. Wang, J. Mater. Chem. A, 2015, 3, 1880918828.

3 J. R. Jennings, Y. Liu, F. Safari-Alamuti and Q. Wang, J. Phys. Chem. C, 2012, 116, 1556-1562.

4 S. A. Berhe, H. B. Gobeze, S. D. Pokharel, E. Park and W. J. Youngblood, ACS Appl. Mater. Interfaces, 2014, 6, 10696-10705.

5 J. Wang and T. Higashihara, Polym. Chem., 2013, 4, 55185526.

6 D. M. Stoltzfus, J. E. Donaghey, A. Armin, P. E. Shaw, P. L. Burn and P. Meredith, Chem. Rev., 2016, 116, 1292012955.

7 J. Wang, C. Lu, T. Mizobe, M. Ueda, W.-C. Chen and T. Higashihara, Macromolecules, 2013, 46, 1783-1793.

8 J. Wang, M. Ueda and T. Higashihara, ACS Macro Lett., 2013, 2, 506-510.

9 J. Wei, G. Ji, C. Zhang, L. Yan, Q. Luo, C. Wang, Q. Chen, J. Yang, L. Chen and C.-Q. Ma, ACS Nano, 2018, 12, 55185529.

10 M. A. Shannon, P. W. Bohn, M. Elimelech, J. G. Georgiadis, B. J. Marinas and A. M. Mayes, Nature, 2008, 452, 301-310.

11 A. Raza, J. Y. Lu, S. Akzaim, H. Li and T. Zhang, Energies, 2018, 11, 253.

12 M. Gao, L. Zhu, C. K. Peh and G. W. Ho, Energy Environ. Sci., 2018, DOI: 10.1039/c8ee01446j.

13 L. Zhu, M. Gao, C. K. N. Peh and G. W. Ho, Mater. Horiz., 2018, 5, 323-343.

14 J. Wang, Y. Li, L. Deng, N. Wei, Y. Weng, S. Dong, D. Qi, J. Qiu, X. Chen and T. Wu, Adv. Mater., 2017, 29, 1603730.

15 Y. Liu, S. Yu, R. Feng, A. Bernard, Y. Liu, Y. Zhang, H. Duan, W. Shang, P. Tao, C. Song and T. Deng, Adv. Mater., 2015, 27, 2768-2774.

16 K. Bar, G. Kang, S. K. Cho, W. Park, K. Kim and W. J. Padilla, Nat. Commun., 2015, 6, 10103.

17 L. Zhou, Y. Tan, J. Wang, W. Xu, Y. Yuan, W. Cai, S. Zhu and J. Zhu, Nat. Photonics, 2016, 10, 393-398.

18 J. Yang, Y. Pang, W. Huang, S. K. Shaw, J. Schiffbauer, A. A. Pillers, X. Mu, S. Luo, T. Zhang, Y. Huang, G. Li, S. Ptasinska, M. Lieberman and T. Luo, ACS Nano, 2017, 11, 5510-5518. 
19 Y. Yang, R. Zhao, T. Zhang, K. Zhao, P. Xiao, Y. Ma, P. M. Ajayan, G. Shi and Y. Chen, ACS Nano, 2018, 12, 829835.

20 X. Hu, W. Xu, L. Zhou, Y. Tan, Y. Wang, S. Zhu and J. Zhu, Adv. Mater., 2017, 29, 1604031.

21 L. Shi, Y. Wang, L. Zhang and P. Wang, J. Mater. Chem. A, 2017, 5, 16212-16219.

22 P. Zhang, Q. Liao, T. Zhang, H. Cheng, Y. Huang, C. Yang, C. Li, L. Jiang and L. Qu, Nano Energy, 2018, 46, 415-422.

23 P. Yang, K. Liu, Q. Chen, J. Li, J. Duan, G. Xue, Z. Xu, W. Xie and J. Zhou, Energy Environ. Sci., 2017, 10, 1923-1927.

24 L. Zhang, B. Tang, J. Wu, R. Li and P. Wang, Adv. Mater., 2015, 27, 4889-4894.

25 N. Xu, X. Hu, W. Xu, X. Li, L. Zhou, S. Zhu and J. Zhu, Adv. Mater., 2017, 29, 1606762.

26 C. Chen, Y. Li, H. Song, Z. Yang, Y. Kuang, E. Hitz, C. Jia, A. Gong, F. Jiang, J. Zhu, B. Yang, J. Xie and L. Hu, Adv. Mater., 2017, 29, 1701756.

27 Y. Fu, G. Wang, X. Ming, X. Liu, B. Hou, T. Mei, J. Li, J. Wang and X. Wang, Carbon, 2018, 130, 250-256.

28 F. Jiang, H. Liu, Y. Li, Y. Kuang, X. Xu, C. Chen, H. Huang, C. Jia, X. Zhao, E. Hitz, Y. Zhou, R. Yang, L. Cui and L. Hu, ACS Appl. Mater. Interfaces, 2018, 10, 1104-1112.

29 Y. Fu, G. Wang, T. Mei, J. Li, J. Wang and X. Wang, ACS Sustainable Chem. Eng., 2017, 5, 4665-4671.

30 P. Mu, W. Bai, Z. Zhang, J. He, H. Sun, Z. Zhu, W. Liang and A. Li, J. Mater. Chem. A, 2018, 6, 18183-18190.

31 H. Ren, M. Tang, B. Guan, K. Wang, J. Yang, F. Wang, M. Wang, J. Shan, Z. Chen, D. Wei, H. Peng and Z. Liu, Adv. Mater., 2017, 29, 1702590.

32 X. Zhou, F. Zhao, Y. Guo, Y. Zhang and G. Yu, Energy Environ. Sci., 2018, 11, 1985-1992.
33 F. Zhao, X. Zhou, Y. Shi, X. Qian, M. Alexander, X. Zhao, S. Mendez, R. Yang, L. Qu and G. Yu, Nat. Nanotechnol., 2018, 13, 489-495.

34 Y. Chang, Z. Wang, Y. Shi, X. Ma, L. Ma, Y. Zhang and J. Zhan, J. Mater. Chem. A, 2018, 6, 10939-10946.

35 J. Wang, R. Du and X. Zhang, ACS Appl. Mater. Interfaces, 2018, 10, 1468-1473.

36 J. Wang, X. Wang and X. Zhang, J. Mater. Chem. A, 2017, 5, 4308-4313.

37 J. Wang and X. Zhang, ACS Nano, 2015, 9, 11389-11397.

38 J. Wang, Y. Zhang, Y. Wei and X. Zhang, Microporous Mesoporous Mater., 2015, 218, 192-198.

39 D. Wen and A. Eychmüller, Chem. Commun., 2017, 53, 12608-12621.

40 C. Ziegler, A. Wolf, W. Liu, A.-K. Herrmann, N. Gaponik and A. Eychmüller, Angew. Chem., Int. Ed., 2017, 56, 13200-13221.

41 J. P. Vareda, A. Lamy-Mendes and L. Duraes, Microporous Mesoporous Mater., 2018, 258, 211-216.

42 C. Zhou, J. Han, G. Song and R. Guo, J. Polym. Sci., Part A: Polym. Chem., 2008, 46, 3563-3572.

43 D. Dong, H. Guo, G. Li, L. Yan, X. Zhang and W. Song, Nano Energy, 2017, 39, 470-477.

44 F. Xu, Z. Tang, S. Huang, L. Chen, Y. Liang, W. Mai, H. Zhong, R. Fu and D. Wu, Nat. Commun., 2015, 6, 7221.

45 J. Wang, Y. Wei, W. He and X. Zhang, RSC Adv., 2014, 4, 51146-51155.

46 J. Wang, Y. Zhang and X. Zhang, J. Mater. Chem. A, 2016, 4, 11408-11415.

47 H. Peng, G. Ma, K. Sun, J. Mu, X. Zhou and Z. Lei, RSC Adv., 2015, 5, 12034-12042.

48 M. S. Zielinski, J.-W. Choi, T. L. Grange, M. Modestino, S. M. H. Hashemi, Y. Pu, S. Birkhold, J. A. Hubbell and D. Psaltis, Nano Lett., 2016, 16, 2159-2167. 